\title{
Hypoactivation of the Ventral and Dorsal Striatum During Reward and Loss Anticipation in Antipsychotic and Mood Stabilizer-Naive Bipolar Disorder
}

\author{
Sarah W Yip ${ }^{*, 1,2}$, Patrick D Worhunsky ${ }^{1,2}$, Robert D Rogers ${ }^{1,3}$ and Guy M Goodwin' \\ 'Department of Psychiatry, University of Oxford, Oxford, UK; ' Department of Psychiatry, Yale University School of Medicine, New Haven, CT, \\ USA; ${ }^{3}$ School of Psychology, Bangor University, Gwynedd, UK
}

\begin{abstract}
Increased activity within known reward-processing neurocircuitry (eg, ventral striatum, VS) has been reported among medicated individuals with bipolar disorder (BD) I and II. However, such findings are confounded by the potential ameliorative effects of moodstabilizing and antipsychotic medications on neural activations. This study tests the hypothesis that a pathophysiological locus of alterations in reward processing is present within the striatum in antipsychotic and lithium-naive individuals with BD. Twenty antipsychotic and lithium-naive individuals with BD II or BD not-otherwise specified (NOS) and 20 matched healthy comparison individuals participated in functional magnetic resonance imaging during the performance of a monetary incentive delay task. Between-group comparisons were conducted using small-volume correction focusing on orthogonal a priori regions of interest centered in the VS and dorsal striatum (DS), respectively. During reward anticipation, unmedicated individuals with BD I/NOS had decreased activity within the DS (but not VS). During loss anticipation, on the other hand, decreased activation within both the VS and DS was observed. Across all participants, DS activity (during reward anticipation) was positively associated with putamen volume. This is the first report of decreased dorsal and ventral striatal activity among unmedicated individuals with BD II/NOS. These data contradict a simple 'reward hypersensitivity' model of $\mathrm{BD}$, and add to a growing body of literature suggesting that blunted reward processing may be a vulnerability factor for both mood- and addiction-related disorders.
\end{abstract}

Neuropsychopharmacology (2015) 40,658-666; doi:I0.1038/npp.2014.2I5; published online 17 September 20I4

\section{INTRODUCTION}

Hypomania and mania are characterized clinically by increase in reward-seeking and impulsive behavior. These experiences define bipolar disorder (BD), and are usually invoked to explain other risky behaviors such as alcohol use disorders (AUDs), other addictions, and suicidality also associated with the disorder (Swann et al, 2005; Merikangas et al, 2007; Di Nicola et al, 2010). However, the neural basis for such behavior is not yet established.

Studies of medicated patients with BD have reported increased activity in the ventral striatum (VS) during reward and affective processing (Lawrence et al, 2004; Nusslock et al, 2012; Caseras et al, 2013), and this has been interpreted as reflecting increased reward sensitivity and more generally a 'reward hypersensitivity model for BD' (Caseras et al, 2013). However, nascent data suggest ameliorative effects of antipsychotic and mood-stabilizing medications on neural activity

\footnotetext{
* Correspondence: Dr SW Yip, Department of Psychiatry, Yale University School of Medicine, I Church Street, Room 732, Suite 7, New Haven, CT 065 I9, USA, Tel: + | 203704 7588, Fax: + | 203737 359|, E-mail: sarah.yip@yale.edu

Received 12 May 20 I4; revised 25 July 20 I4; accepted I 3 August 20 I4; accepted article preview online 20 August 2014
}

within limbic circuits, making accurate interpretation of data from medicated individuals difficult (Haldane et al, 2008; Phillips et al, 2008; Passarotti et al, 2011). Thus, studies of unmedicated young adults either at risk for BD or early in the disease course are needed to test the hypothesis of a pathophysiological locus of reward-processing alterations in BD (Nusslock et al, 2012). Such an assessment is important from both a clinical and a research perspective, and is consistent with the increasing emphasis within psychiatry on identifying common and distinct biomarkers for disorders (eg, Research Domain Criteria) (Insel et al, 2010).

We previously demonstrated a reduced subjective psychostimulant response to acute ethanol administration among young men at increased risk for BD (Yip et al, 2012), which-as highlighted by Schuckit (2012) —was strikingly similar to the 'low level response' frequently observed among young adults family history positive for AUDs (eg, Schuckit and Smith, 1996; Schuckit, 2012). This low level of response in completely antipsychotic-naive subjects could reflect neurodevelopmental alterations-eg, in dopaminergic transmission (Bahi and Dreyer, 2012) or in white matter pathway development (Yip et al, 2013)relating to the elevated rates of both substance and nonsubstance addictions observed across BD subtypes (Swann et al, 2005; Merikangas et al, 2007; Di Nicola et al, 2010; 
Swann, 2010). From this perspective, altered neural processing of rewards may be a shared risk-factor between $\mathrm{BD}$ and addictive disorders (Yip et al, 2012). In the present study, we therefore tested the hypothesis of altered striatal activity among euthymic, antipsychotic- and mood stabilizer-naive young adults with $\mathrm{BD}$.

Monetary incentive delay (MID) tasks allow for dissociation of reward anticipation and reward outcome processing, independent of decision making (Knutson and Greer, 2008; Andrews et al, 2011). Given the well-documented alterations in risk-reward decision making observed among both medicated and unmedicated individuals with BD across mood states (Chandler et al, 2009; Adida et al, 2011), assessment of reward-related neurocircuity in the absence of a decision-making component is particularly important for studies in this population. We chose to use a modified MID task, previously used to study reward processing among individuals family history positive for AUDs (Andrews et al, 2011).

Using the modified MID task, Andrews et al (2011) reported blunted VS activity during reward anticipation among individuals family history positive for AUDs, suggesting that neural alterations in reward processing may be a vulnerability marker for AUDs. Thus, based on our previous findings of blunted responses to acute ethanol among unmedicated individuals with high rates of hypomania (Yip et al, 2012), we hypothesized blunted VS activity during reward anticipation and outcome processing among euthymic-, antipsychotic-, and mood stabilizer-naive young adults with BD II/NOS.

Previous studies of reward processing in both $\mathrm{BD}$ and addictions have generally focused on the ventral component of the striatum, however a growing body of literature also implicates the dorsal striatum (DS) in the neurobiology of substance- and mood-related disorders (Vollstadt-Klein et al, 2010; Hyatt et al, 2012; Moses-Kolko et al, 2012). Thus, a further aim of this study was to assess neural activations within both the dorsal and ventral components of this region. Finally, given a recent report of a positive association between putamen volumes and VS activity during reward anticipation among medicated individuals with BD (Caseras et al, 2013), we further planned to explore this relationship in our unmedicated sample of young adults with BD II/NOS.

\section{MATERIALS AND METHODS}

\section{Participants and Screening}

Participants were 20 right-handed individuals with BD II/ NOS and 20 age-, gender-, and cognitive ability-matched right-handed healthy control (HC) participants. Participants were recruited from the University of Oxford and the surrounding community. All participants were screened and underwent functional and structural imaging protocols at the University of Oxford Centre for Clinical Magnetic Resonance Research. The experimental protocol was approved by the National Health Service Research Ethics Committee. Following complete description of the study's procedures, all participants provided written informed consent.
All participants were screened using the Mini-International Neuropsychiatric Interview (MINI) (Sheehan et al, 1998). In addition to previous experiences of hypomania (as defined using the DSM-IV-TR), all participants had experienced depressive symptoms in the past; however, for a subset of participants depressive episodes did not meet the 2-week criteria for major depression. As such, these individuals were classified as $\mathrm{BD} / \mathrm{NOS}(n=5)$, rather than BD II. Exclusion criteria for BD II/NOS participants were as follows: (i) any current psychiatric disorder (with the exception of BD and anxiety disorders); (ii) current major depressive, manic, or hypomanic episodes at the time of scanning (as assessed using the MINI); (iii) any current psychotropic medication; (iv) any past treatment with an antipsychotic or mood stabilizer (eg, lithium and anticonvulsants); (v) any history of head injury or neurological condition; (vi) any other contraindication to scanning. Four of the BD II/NOS participants met criteria for past alcohol abuse, two met criteria for past marijuana abuse, and one met criteria for past codeine abuse and past anorexia. Three BD II/NOS participants had received previous SSRI treatment, and all had been medication-free for a minimum of 3 months prior to scanning.

Exclusion criteria for HC participants were as follows: (i) endorsement of any previous hypomanic symptoms, as defined using the Mood Disorders Questionnaire (Hirschfeld et al, 2000); (ii) any current or past psychiatric disorder, as assessed using the MINI; (iii) previous exposure to psychotropic medication; (iv) any history of head injury or neurological condition; (v) any other contraindication to scanning.

Participants also completed psychometric assessments of cognitive ability (Raven's Matrices) (Raven et al, 1998), current depressive symptoms (Hamilton Depression Rating Scale, HAM-D) (Hamilton, 1960), current bipolar symptoms (Young Mania Rating Scale) (Young et al, 1978), impulsivity (Barratt Impulsiveness Scale) (Patton et al, 1995), and state and trait affect (Positive and Negative Affect Scales) (Watson et al, 1988).

\section{MID Task}

The modified version of the MID task has been described previously (Andrews et al, 2011) and is shown in Supplementary Figure 1. Briefly, each trial consists of three stages. First, participants were presented with one of six cues for 1000 ms (WIN $\$ 0$, WIN \$1, WIN \$5, LOSE \$0, LOSE \$1, and LOSE $\$ 5$ ), followed by a fixation cross (prospect of reward/ loss phase, A1). Participants then waited for the presentation of a subsequent target stimulus before responding with a single button press as quickly as possible. Participants then fixated on a cross (anticipation of reward/loss phase, A2) before receiving feedback on the outcome of the trial (eg, YOU WON \$1; YOU DID NOT WIN \$1; YOU LOST \$1; YOU DID NOT LOSE \$1).

Thus, the task includes six distinct events of interest: (i) processing the prospect of possible rewards (ie, 'gain prospects'; A1, win trials); (ii) processing the prospect of possible losses (ie, 'loss prospects'; A1, loss trials); (iii) the anticipation of reward (ie, 'gain anticipation'; A2, win trials); (iv) the anticipation of loss (ie, 'loss anticipation'; A2, loss trials); (v) the delivery of reward outcomes (ie, 'gain 
outcomes'); and (vi) the delivery of loss outcomes (ie, 'loss outcomes'). The task also includes appropriate neutral comparison events for all phases (eg, anticipation of winning $\$ 0$ ). The total completion time for the MID is 12 min (22 win trials, 22 loss trials, and 11 neutral trials). Each participant played the task twice.

\section{Individual Calibration of Target Stimuli}

As has been reported previously (Andrews et al, 2011), the durations of the target stimuli were individually calibrated for each participant based on their performance during a brief practice session outside of the scanner. This calibration was conducted to ensure that all participants experienced relatively equivalent numbers of hits (eg, YOU WON $\$ 1$ or YOU DID NOT LOSE \$1) and misses (eg, YOU DID NOT WIN \$1 or YOU LOST \$1), independent of variability in reaction times. Specifically, the calibration aims to achieve a mean hit rate of $67 \%$, meaning that on two-thirds of win trial participants will hit the target and receive a reward (eg, YOU WON \$1), but will fail to hit the target and therefore not receive a reward on one-third of win trials (eg, YOU DID NOT WIN \$1). Similarly, participants will hit the target and successfully avoid a loss on two-thirds of loss trials (eg, YOU DID NOT LOSE \$1), but will fail to hit the target and will receive a loss on one-third of the trials (eg, YOU LOST \$1). As presented in Table 1, in actuality, the HC participants had a mean hit rate of $71 \%$ and $\mathrm{BD}$ II/NOS participants had a mean hit rate of $69 \%$. Whereas these hit rates are slightly higher than the optimum rate of $67 \%$, they are nonetheless comparable to hit rates in other studies using this calibration method (Andrews et al, 2011), and did not differ significantly between participant groups, meaning that the average number of reward (loss) events did not differ between HCs and BD II/NOS participants.

\section{Image Acquisition}

Images were acquired using a Siemens Trio $3 \mathrm{~T}$ scanner and a $\mathrm{T} 2^{\star}$-sensitive echo-planar image gradient echo pulse sequence (repetition time/echo time (TR/TE) $1500 / 27 \mathrm{~ms}$, flip angle $60^{\circ}$, field of view $220 \mathrm{~mm} \times 220 \mathrm{~mm}, 64 \times 64$ data matrix, $3.4 \mathrm{~mm} \times 3.4 \mathrm{~mm}$ in-plane resolution, slice thickness $4 \mathrm{~mm}$ with $1 \mathrm{~mm}$ skip, $5 \mathrm{~mm}$ effective slice thickness, and 25 slices).

\section{Analyses}

Demographic, clinical, and behavioral characteristics. Between-group comparison of demographic, clinical characteristics, and behavioral task performance measures were conducted using one-way analyses of variance (ANOVAs), Mann-Whitney $U$-tests (reaction times), and chi-square tests (gender) as appropriate.

\section{Functional Image Analyses}

Preprocessing. Spatial preprocessing was conducted using SPM8 (Wellcome Functional Imaging Laboratory, London, UK). All functional scans were realigned separately before normalization to Montreal Neurological Institute (MNI)
Table I Demographic, Clinical, and Behavioral Characteristics of Healthy Control $(\mathrm{HC})$ and Bipolar Disorder II/Not-OtherwiseSpecified Disorder (BD II/NOS) Participants

\begin{tabular}{|c|c|c|c|c|}
\hline & \multicolumn{2}{|c|}{$\mathrm{HC}(n=20)$} & \multicolumn{2}{|c|}{ BD $(n=20)$} \\
\hline & $n$ & $\%$ & $n$ & $\%$ \\
\hline BD subtype (BD II) & - & - & 15 & 75 \\
\hline \multirow[t]{2}{*}{ Gender (male) } & 10 & 50 & 12 & 60 \\
\hline & Mean & SE & Mean & SE \\
\hline Age & 22.1 & 0.58 & 22.59 & 0.90 \\
\hline Cognitive ability & 3.37 & 0.65 & 4.00 & 0.95 \\
\hline $\mathrm{MDQ}$ & - & - & 10.12 & 0.41 \\
\hline BIS-II & 53.79 & 1.67 & 74.71 & 1.49**** \\
\hline YMRS & 0.05 & 0.05 & 1.24 & $0.44 * *$ \\
\hline HAM-D & 3.21 & 0.72 & 9.24 & $1.54 * * * *$ \\
\hline PPANAS trait & 32.94 & 1.35 & 33.00 & 1.66 \\
\hline NPANAS trait & 13.89 & 0.96 & 20.65 & $2.09 * *$ \\
\hline PPANAS state & 28.26 & 1.63 & 29.63 & 1.96 \\
\hline NPANAS state & 11.79 & 0.63 & $\mid 3.31$ & 0.69 \\
\hline \multicolumn{5}{|l|}{ MID performance } \\
\hline \multirow[t]{2}{*}{ Hit rate (\%) } & 71.27 & 2.83 & 69.32 & 2.33 \\
\hline & Median & SE & Median & SE \\
\hline Reaction time (ms) & 205.49 & 28.43 & 205.8 & 63.35 \\
\hline Co-occurring MINI diagnoses & Current & Past & Current & Past \\
\hline Suicidality (low risk) & - & - & I & $\mathrm{n} / \mathrm{a}^{\mathrm{a}}$ \\
\hline Panic & - & - & I & 5 \\
\hline Agoraphobia & - & - & 0 & 2 \\
\hline Social anxiety disorder & - & - & 2 & 0 \\
\hline OCD & - & - & 3 & 0 \\
\hline Specific phobia & - & - & । & 0 \\
\hline GAD & - & - & 3 & 0 \\
\hline Hypochondriasis & - & - & 2 & 0 \\
\hline Alcohol abuse & - & - & 0 & 4 \\
\hline Substance abuse ${ }^{b}$ & - & - & 0 & 3 \\
\hline Anorexia & - & - & 0 & I \\
\hline \multicolumn{5}{|l|}{ Psychotropic medication } \\
\hline SSRIs & - & - & 0 & 3 \\
\hline \multicolumn{5}{|c|}{$\begin{array}{l}\text { Abbreviations: BIS-I I, Barratt Impulsiveness Scale; GAD, generalized anxiety } \\
\text { disorder; HAM-D, Hamilton Depression Inventory; MDQ, Mood Disorders } \\
\text { Questionnaire; OCD, obsessive-compulsive disorder; PANAS, Positive and } \\
\text { Negative Affect scales; SSRI, selective serotonin reuptake inhibitor; } \\
\text { YMRS, Young Mania Rating Scale. } \\
{ }^{\mathrm{a}} \mathrm{n} / \mathrm{a}=\text { not assessed by the MINI. } \\
{ }^{\mathrm{b}} \text { Two participants had past marijuana abuse; one participant had past codeine } \\
\text { abuse. } \\
{ }^{*} * * * * 0.00 \text { I; } * * * 0.0 \text { I. }\end{array}$} \\
\hline
\end{tabular}

standard space (voxel size $=3 \times 3 \times 3 \mathrm{~mm}^{3}$ ). Scans with participant motion in excess of one voxel were excluded. Data were smoothed with a 6-mm full-width-half-maximum Gaussian kernel. 


\section{Region-of-Interest (ROI) Analyses}

Data analysis was conducted using previously described methodology (Jia et al, 2011). Consistent with Knutson and Greer (2008), all events of interest were contrasted to their analogous neutral phase (single-subject level statistics). For example, during the prospect of reward (A1) phase, gain prospects (eg, WIN \$1) were compared with 'no gain' prospects (WIN \$0). As in previous studies using the modified MID task-(Jia et al, 2011; Balodis et al, 2012; Patel et al, 2013) - group-level random-effects models (twotailed $t$-tests in SPM8) using these contrasts were then conducted to explore any significant between-group differences in BOLD response during the prospect of reward/loss (A1), anticipation of reward/loss (A2), and outcome of reward/loss phases. Consistent with previous studies (Jia et al, 2011; Balodis et al, 2012; Patel et al, 2013), only 'actual' wins (eg, 'WON \$1) and 'actual' losses (eg, 'LOST \$1) were included for the outcome phase. Therefore, in order to reduce excess noise associated with a small number of events of interest, $\$ 1$ and $\$ 5$ trials were combined and compared with $\$ 0$ (neutral control trials) for all phases. All ROI analyses focused on the ventral and dorsal components of the striatum, as defined using previously identified coordinates, and were conducted using the small-volume correction (SVC) tool in SPM8; $p$-values were thresholded using family-wise error correction $(p \mathrm{FWE}<0.05)$. Coordinates for the VS were taken from meta-analytic data of previous functional magnetic resonance imaging (fMRI) studies of MID task performance (left: $x=-12, y=10$, $z=-2$; right: $x=10, y=8, z=2$ ) (Knutson and Greer, 2008), consistent with other recent studies using this version of the MID (eg, Balodis et al, 2012). The DS was defined using coordinates from published fMRI data indicating activation of the DS in response to a range of different rewarding stimuli (left: $x=-9, y=3, z=15$; right: $x=9, y=3, z=15$ ) (Valentin and O'Doherty, 2009), consistent with other fMRI studies focusing on the DS (eg, Jessup and O'Doherty, 2011).

\section{Whole-Brain Analyses Exploring Magnitude and Valence Effects}

Whole-brain analyses were conducted as exploratory investigations of task effects and comparisons of cases and controls. Multi-level ANOVAs were constructed using the 'Flexible Factorial' utility in SPM8 in order to explore the effects of different task-related variables on whole-brain activations. For the $\mathrm{A} 1$ and $\mathrm{A} 2$ phases, these included the between-subjects factor of group (HC/BD) and the withinsubjects factors of reward valence (win/lose) and reward magnitude $(\$ 1 / \$ 5)$. As described above, non-winning (eg, 'DID NOT WIN \$1') and non-losing (eg, 'DID NOT LOSE $\$ 1$ ') events were excluded from analyses during the outcome phase. This allows for assessment of absolute effects of winning and losing (Patel et al, 2013); however, this also limits statistical power as it reduced the total number of events of interest. Therefore, for the outcome phase (as in our primary ROI analyses and consistent with previous studies (eg, Patel et al, 2013), $\$ 1$ and $\$ 5$ trials were combined and compared against $\$ 0$ trials. Whole- brain statistical maps were corrected at $p \mathrm{FWE}<0.05$ $(k>50)$.

\section{Structural Analysis of the Putamen}

Whole-brain volumetric analyses were conducted previously as part of a larger, published study of white and gray matter structural characteristics of unmedicated BD (Yip et al, 2013) using whole-brain voxel-based morphometry (VBM), as implemented in FSL. In order to explore the association between striatal activations and putamen volumes in this study, individual gray matter volumes within the putamen were extracted using fsl_roi (see Supplementary Materials for further details on structural analyses).

\section{Correlational and Substance Use Analyses}

Individual peak signals within the VS and DS (as identified in the SVC analyses) were extracted using 'measure_roi_ val_spm8' and these were entered into SPSS for subsequent correlational analysis with putamen volumes. Further, in order to determine the putative effects of residual depressive symptoms on the observed neural activations, correlational analyses with HAM-D scores were additionally conducted. All correlations were conducted using Pearsons's $r$, and considered significant at two-tailed $(p<0.05)$.

Several of the BD II/NOS participants in this study had a history of previous alcohol $(n=4)$ or other substance $(n=3)$ abuse. Thus, post hoc comparisons excluding these individuals were conducted using the peak activations identified in the SVC analyses in order to test whether the between-group differences in activations within the VS and DS during the A2 period remained after excluding individuals with a previous history of alcohol or other drug abuse.

\section{RESULTS}

\section{Demographic, Clinical, and Behavioral Characteristics}

Demographic and clinical characteristics of the BD II/NOS and HC participants are shown in Table 1. There were no significant between-group differences in gender, age, cognitive ability, trait-positive affect, and state-positive or -negative affect $(\mathrm{Fs}<2.66, p$ 's $>0.11)$. As anticipated, BD II/NOS participants reported significantly more current hypomanic symptoms $(\mathrm{F}=7.90, p=0.008)$, trait-negative affect $(\mathrm{F}=8.95, p=0.005)$, and trait impulsivity $(\mathrm{F}=85.84$, $p<0.001)$. As in previous studies of euthymic BD I and II (Caseras et al, 2013), HAM-D scores were elevated among the BD II/NOS participants in comparison with controls $(\mathrm{F}=13.50, p=0.001)$, indicating the presence of some residual depressive symptoms within the past month. All participants were confirmed euthymic at the time of scanning using the MINI (structured clinical interview).

\section{MID Task Results}

Performance. No significant differences in mean reaction times or hit rate $(p$ 's $>0.5)$ were observed between BD II/NOS and HC participants (Table 1). 
Table 2 Findings from Small-Volume Corrected Group-Level Random-Effects Models Focusing on the Dorsal and Ventral Striatum (pFWE<0.05)

\begin{tabular}{|c|c|c|c|c|c|}
\hline \multirow[t]{2}{*}{ Phase } & \multicolumn{3}{|c|}{ Peak MNI } & \multirow[t]{2}{*}{$t$-value } & \multirow[t]{2}{*}{ pFWE } \\
\hline & $x$ & $y$ & $\mathbf{z}$ & & \\
\hline \multicolumn{6}{|c|}{ Dorsal striatum } \\
\hline \multicolumn{6}{|c|}{ A2 Wins } \\
\hline Left & -15 & -1 & 10 & -2.14 & $>0.10$ \\
\hline Right & 9 & 5 & 13 & -3.28 & $<0.05$ \\
\hline \multicolumn{6}{|c|}{ A2 Losses } \\
\hline Left & -15 & 2 & 16 & -2.27 & $>0.10$ \\
\hline Right & 9 & 5 & 13 & -3.31 & $<0.05$ \\
\hline \multicolumn{6}{|c|}{ Ventral striatum } \\
\hline \multicolumn{6}{|c|}{ A2 Wins } \\
\hline Left & -12 & 2 & I & -1.44 & $>0.10$ \\
\hline Right & 9 & 5 & 10 & -2.94 & $<0.10$ \\
\hline \multicolumn{6}{|c|}{ A2 Losses } \\
\hline Left & -6 & 11 & -8 & -1.10 & $>0.10$ \\
\hline Right & 15 & 2 & 4 & -3.40 & $<0.05$ \\
\hline
\end{tabular}

Abbreviation: FWE, family-wise error corrected.

The dorsal and ventral striatum ROls were defined using $9 \mathrm{~mm}$ spheres centering on the following coordinates: dorsal ROls: left: $x=-9, y=3, z=15$; right: $x=9, y=3, z=15$; ventral ROls: left: $x=-12, y=10, z=-2$; right: $x=10, y=8, z=2$. Negative $t$-values indicate $B D<H C$

\section{Neural Responses}

VS and DS. Findings from between-group comparisons of neural activations during the A2 phase are shown in Table 2 and in Figure 1. In comparison with matched controls, individuals with $\mathrm{BD}$ II/NOS had significantly decreased activity within the right DS during reward anticipation (A2 phase; $t=-3.279, p \mathrm{FWE}=0.046)$, but did not differ significantly from controls in VS activity $(t=-2.944, p \mathrm{FWE}=0.089)$ during this phase. During loss anticipation, BD II/NOS participants exhibited diminished right VS and right DS activity (A2 phase; $t=-3.396, p \mathrm{FWE}=0.038 ; t=-3.306, p \mathrm{FWE}=$ 0.047; Figure 1) in comparison with controls. No significant between-group differences were observed within these a priori ROIs during any other phases of the MID ( $p$ FWE $>0.05)$.

\section{Relationship to Putamen Volumes}

Consistent with our previously published data (Yip et al, 2013), there were no between-group differences in putamen volumes between control and BD II/NOS participants. Across all participants, there was a significant positive association between activity within the DS during reward anticipation (A2 phase) and right putamen volumes (Figure 2; $r=0.35, p=0.03$ ).

\section{Relationship to Depression Scores and Substance Use History}

No significant associations between BOLD responses and HAM-D scores were observed within the BD II/NOS or HC groups ( $p$ 's $>0.25$ ). After excluding BD II/NOS participants with a previous history of alcohol or substance abuse $(n=7)$, the previously reported between-group differences in BOLD signals within the VS and DS during the A2 period remained significant $(p$ 's $<0.01)$.

\section{Whole-Brain Analyses}

Detailed cluster information for all of the significant main and interaction effects examined in the multi-level flexible factorial model is shown in Supplementary Table 1.

Results of the flexible factorial model indicated no significant main or interaction effects of diagnostic group on whole-brain BOLD responses ( $p$ FWEs $>0.05$ ) for any of the three task phases. Results of the flexible factorial model indicated a significant main effect of reward valence (wins vs losses) during the $\mathrm{A} 1$ phase within a cluster encompassing regions of the cuneus, lingual gyrus, and primary and secondary visual cortices $(\mathrm{F}=85.06, p \mathrm{FWE}<0.001)$. There were no significant main or interaction effects of reward valence on whole-brain activations during the A2 or outcome phases.

Results of the flexible factorial model indicated significant main effects of reward magnitude ( $\$ 5 v s \$ 1$ ) during the A1 phase within six clusters. The first cluster included regions of the caudate, putamen, thalamus, midbrain, inferior frontal gyrus, and insula $(\mathrm{F}=90.28, p \mathrm{FWE}<0.001)$. The second cluster included regions of the anterior cingulate, cingulate gyrus, medial frontal gyrus, cingulum, primary motor cortex, supplementary motor area (SMA), middle frontal gyrus, and superior frontal gyrus $(\mathrm{F}=73.94$, $p \mathrm{FWE}<0.001)$. The third cluster encompassed regions of the precuneus, cuneus, primary and secondary visual cortices, and the somatosensory association cortex $(\mathrm{F}=48.72, p \mathrm{FWE}<0.001)$. The fourth cluster primarily included a region of the right precuneus $(\mathrm{F}=45.12$, $p \mathrm{FWE}<0.001)$. The fifth cluster included regions of the middle frontal gyrus, SMA, and precentral gyrus $(\mathrm{F}=37.32$, $p F W E<0.001)$, and the sixth cluster encompassed parts of the anterior and posterior cerebellum $(\mathrm{F}=37.02$, $p \mathrm{FWE}<0.001)$. During the outcome phase, there was a significant main effect of reward magnitude ( $\$ 0$ vs $\$ 1 / \$ 5$ ) in a single cluster encompassing part of the anterior cingulate cortex $(\mathrm{F}=45.46, p \mathrm{FWE}<0.001)$. There were no significant main or interaction effects of reward magnitude on whole-brain activations during the A2 period $(p$ FWEs $>0.05)$.

\section{DISCUSSION}

Consistent with our primary hypothesis, young adults with BD II/NOS showed reduced BOLD amplitudes within the striatum during the anticipation phase (A2) of the modified MID task (Andrews et al, 2011) for both wins and losses. Taken together with our previous findings of a reduced subjective response to acute ethanol among young adults with high rates of hypomanic experiences (Yip et al, 2012) - in conjunction with fMRI data demonstrating decreased BOLD response during the A2 phase among individuals family history positive for AUDs (Andrews $\mathrm{et} \mathrm{al}$, 2011) - these data suggest that blunted experiences of 


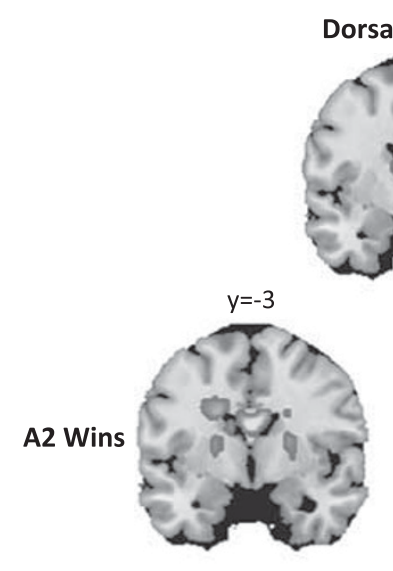

Dorsal Striatum
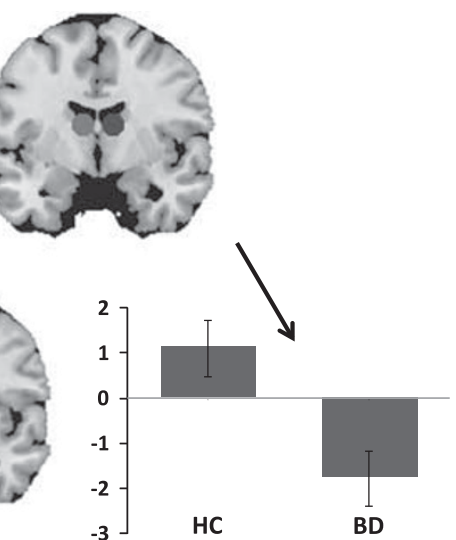

A2 Losses
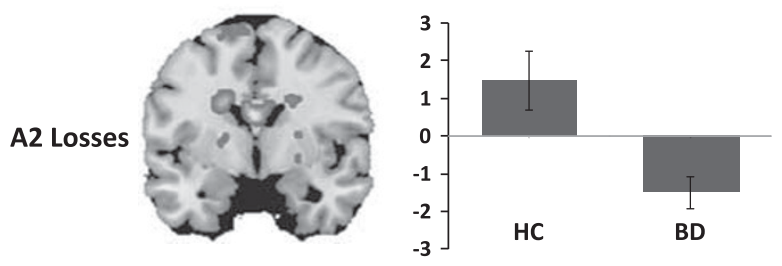
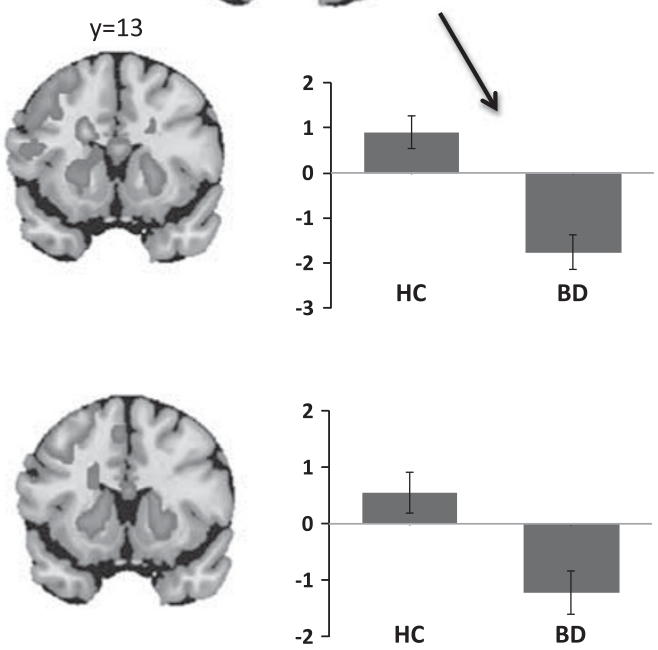

Figure I Reduced dorsal and ventral striatal activations during gain and loss anticipation (A2 phase of the MID task). Whole-brain, task-related activations ( $k>50 ; p<0.00 \mathrm{I}$, uncorrected) across all participants are shown in coronal slices. Dorsal and ventral ROls are shown in red (dorsal) and blue (ventral), and average peak activations are plotted for $\mathrm{HC}$ and BD II/NOS groups. Group-level random-effects models were conducted using the small-volume correction (SVC) tool in SPM8, and p-values were thresholded using family-wise error correction (pFWE <0.05). These analyses indicated significantly decreased activity among BD II/NOS participants (vs controls) in the dorsal striatum during win anticipation, and within both the dorsal and ventral striatum during loss anticipation. BD, bipolar II or not-otherwise-specified disorder; HC, healthy control.

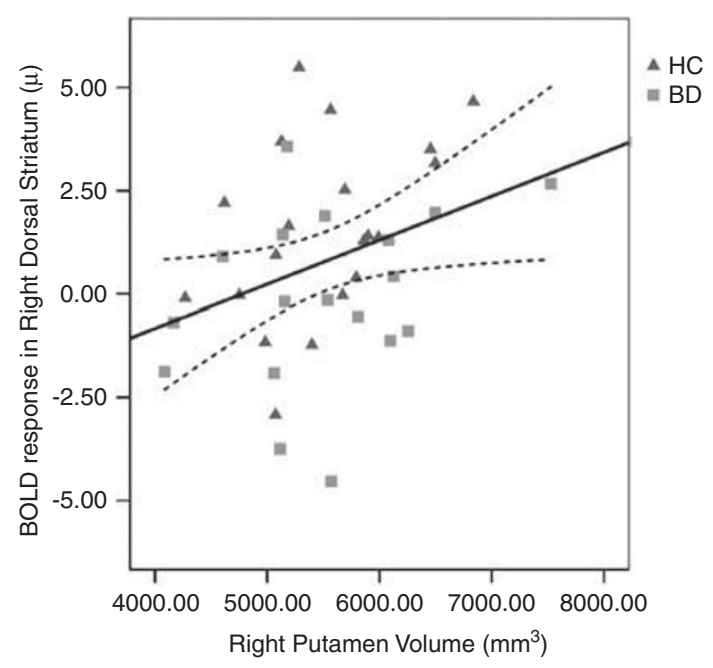

Figure 2 Correlation between putamen volumes and BOLD response (shown in $u=$ arbitrary units) within the right dorsal striatum ( $9 \mathrm{~mm} \mathrm{ROI}$ ) during the anticipation of winning outcomes. BD, bipolar II or nototherwise-specified disorder; HC, healthy control.

reward may be a vulnerability factor for both mood and alcohol use disorders.

None of the BD participants included in this study had been treated with antipsychotics or mood stabilizers, and all were early in the disease course-hence, the observed differences cannot be attributed to the effects of medication or the consequences of prolonged illness. Our findings differ from those from studies of largely medicated individuals, and are contrary to a 'reward hypersensitivity' model of BD (Caseras et al, 2013) - at least in so far as this might predict increased BOLD responses within the striatum. These differences highlight the importance of assessing neural function across different subpopulations within a single disorder. Our data nonetheless add to the growing body of literature implicating striatal function in the pathophysiology of BD II-eg, Caseras et al (2013) which may itself be an important replication.

To our knowledge, this is the first study of reward processing in $\mathrm{BD}$ to focus on both the dorsal and ventral components of the striatum. The VS has long been implicated in the processing of both rewarding and aversive stimuli and decreased BOLD response within the VS has been identified as a putative biological marker for vulnerability to substance use in adolescents (Peters et al, 2011). Similarly, data implicate the DS in the neurobiology of both mood- and substance-related disorders (Vollstadt-Klein et al, 2010; Hyatt et al, 2012; Dager et al, 2013; Gabbay et al, 2013; Tomasi and Volkow, 2013; Xu et al, 2014). The DS is further involved in reward-related learning and is thought to have a role in substance misuse and addictions relapse (Everitt and Robbins, 2005; Volkow et al, 2006). As such, our findings of decreased responses within both of these components may relate to the elevated rates of alcohol and other substance use among individuals with $\mathrm{BD}$ (Merikangas et al, 2007). 
We used the modified MID task as it requires no choice or guessing by subjects (consistent with the original developed by Knutson and colleagues), making it a relatively pure test of anticipation and reward processing independent of decision making (Knutson and Greer, 2008), which may offer advantages when studying populations characterized by decision-making impairments (Chandler et al, 2009; Adida et al, 2011). Working memory deficits have similarly been consistently documented in BD (Bourne et al, 2013), hence we selected the modified version of the MID task for its use of explicit cues rather than symbols (thus eliminating any unnecessary working memory involvement). This version further differs from that originally developed by Knutson and Greer (2008), as anticipatory reward processing is separated into reward prospect and reward anticipation phases (Andrews et al, 2011).

Partially consistent with previous findings (Caseras et al, 2013), we found a significant association between BOLD responses within the striatum during monetary reward anticipation and putamen volumes; however this association was observed for activation within the dorsal-rather than ventral-component of the striatum. Together with the findings from Caseras et al (2013), these data underscore the need for further research into the relationship between functional and structural neural characteristics in BD.

\section{Whole-Brain Findings}

To our knowledge, this is the first study to utilize exploratory, whole-brain analyses including the task-related variables of reward magnitude and reward valence as within-subjects factors and diagnosis as the betweensubjects factor during performance of the modified version of the MID task (Andrews et al, 2011). During the A1 and outcome phases, robust effects of reward magnitude were observed across multiple brain regions previously implicated in reward processing and value encoding (eg, caudate, putamen, midbrain, thalamus, insula, and anterior cingulate); however, there were no significant main or interaction effects of diagnosis. No significant whole-brain effects during the A2 phase were found for any of the variables examined using this model.

Previous studies conducted in healthy populations using versions of the MID with more total trial types that do not model the A1 and A2 phases separately have demonstrated significant effects of reward magnitude and reward valence on BOLD responses during reward anticipation; eg, (Samanez-Larkin et al, 2007, 2008). It is likely that our sample size ( $n=20$ per group) was insufficiently powered to detect diagnostic effects during these phases using this type of multi-level model. Therefore, future studies comparing cases and controls using larger sample sizes and/or tasks with more trials per event type to assess magnitude-related factors such as expected value encoding are needed to assess these factors in unmedicated BD (Bermpohl et al, 2010).

\section{Strengths and Limitations}

Most of the previous fMRI studies in BD have included a majority of medicated individuals (Chen et al, 2011). In recent years this has been highlighted as a significant confound in the literature (eg, Nusslock et al, 2012; Caseras et al, 2013), and an increasing number of studies have sought to control for the effects of medication by conducting post hoc comparisons between various medicationbased subgroups. These measures can never be a satisfactory substitute for recruiting drug-free patients, nor do they necessarily address the confounding factor of previous exposure to mood stabilizer and antipsychotic medications (Hafeman et al, 2012). Several recent studies looking at the longitudinal effects of a typical antipsychotics on BOLD signal responses suggest normalizing effects of medication (eg, Haldane et al, 2008; Passarotti et al, 2011). However, no previous studies of reward processing in $\mathrm{BD}$ have controlled for previous medication exposure, despite evidence suggesting partially ameliorative effects of medications on limbic circuits among individuals with BD (Phillips et al, 2008).

However, the practical difficulty of recruiting medicationnaive individuals with $\mathrm{BD}$ is immense. Accordingly, as in previous studies, we recruited BD II and NOS participants early in their illness course and before significant clinical intervention (Chandler et al, 2009; Yip et al, 2013; Godlewska et al, 2014; Yip et al, 2014). A limitation of this methodology is that it inherently excludes very severe cases and may therefore underestimate the magnitude of observed effects. This limitation must be weighed against the benefits of conducting neurobiological investigations in the absence of the previously mentioned confounds. Moreover, it is difficult to see how psychiatry progresses without moving the focus for psychopathology earlier in the illness course, when the potential for disease modification may be greatest. It is likely also to be one of the ways in which we have to break down or refine categorical diagnosis in the future (Insel et al, 2010).

Subsyndromal-depressive symptoms are the rule in euthymic bipolar populations (eg, Bourne et al, 2013), thus the challenge of how to control for residual episodic symptoms in case control studies of $\mathrm{BD}$ remains an ongoing problem within this field. Whereas all of the BD II/NOS participants in this study were judged euthymic on the basis of structured interview (and the DSM-IV 2-week time-frame for a current depressive episode), these participants had some residual depressive symptoms. Our correlational analyses indicated no significant associations between depression scores and neural responses within the DS or VS during reward or loss anticipation among $\mathrm{BD}$ or $\mathrm{HC}$ participants, so our findings cannot be accounted for by the presence of residual depressive symptoms.

A final limitation of this study is that our primary analyses used multiple separate $t$-tests to explore BOLD responses between our two groups. This is justified here by a clear a priori hypothesis and a difficult to recruit bipolar population of finite size. Our overall statistical approach is also consistent with that from other recent studies with strong a priori hypotheses using this version of the MID task to study neural responses in clinical populations (eg, Jia et al, 2011; Balodis et al, 2012; Patel et al, 2013). However, as the field progresses it will be important to use more sophisticated, higher-level models such as mixedeffects ANOVAs (as in our exploratory analyses including magnitude and valence) to explore the interaction effects between the different aspects of task design on neural responses. Such future studies comparing cases and controls will likely require larger sample sizes and/or tasks 
including a larger number of events of interest to ensure sufficient power, but will be important to clarify the relationship between different task-related factors and the functional neurobiology of BD.

\section{CONCLUSIONS AND FUTURE DIRECTIONS}

These data indicate decreased striatal activations during reward and loss anticipation among antipsychotic- and mood stabilizer-naive young adults with BD II/NOS, and offer further evidence of reward system involvement in the pathophysiology of BD. Taken together with previous findings (Andrews et al, 2011), these data further suggest neurofunctional similarities between the individuals at increased risk AUDs and young adults with BD II/NOS, which may account for the elevated co-occurrence rates of the two disorders. The young age and limited illness history of the $\mathrm{BD}$ participants suggests that these alterations may be neurodevelopmental in nature and relate to factors such as an altered balance in catecholamine function or the development of neuronal connections (Yip et al, 2013).

\section{FUNDING AND DISCLOSURE}

This work was funded by the University of Oxford and a PhD Studentship (SWY) from the Medical Research Council (UK). GMG holds or has held grants from Bailly Thomas charity, Medical Research Council, NIHR, Servier; has received honoraria from AstraZeneca, Eli Lilly, GSK, Lundbeck, Otsuka, and Servier, holds shares in P1vital; has served on advisory boards for Cephalon, Lundbeck, Merck, Otsuka, Servier, Takeda, and acted as an expert witness for Eli Lilly. RDR holds a consultancy agreement with Pfizer. The remaining authors declare no conflict of interest.

\section{ACKNOWLEDGEMENTS}

We thank Drs Godfrey D Pearlson and Marc N Potenza for provision of the modified MID task.

\section{REFERENCES}

Adida M, Jollant F, Clark L, Besnier N, Guillaume S, Kaladjian A et al (2011). Trait-related decision-making impairment in the three phases of bipolar disorder. Biol Psychiatry 70: 357-365.

Andrews MM, Meda SA, Thomas AD, Potenza MN, Krystal JH, Worhunsky $\mathrm{P}$ et al (2011). Individuals family history positive for alcoholism show functional magnetic resonance imaging differences in reward sensitivity that are related to impulsivity factors. Biol Psychiatry 69: 675-683.

Bahi A, Dreyer J-L (2012). Involvement of nucleus accumbens dopamine D1 receptors in ethanol drinking, ethanol-induced conditioned place preference, and ethanol-induced psychomotor sensitization in mice. Psychopharmacol 222: 141-153.

Balodis IM, Kober H, Worhunsky PD, Stevens MC, Pearlson GD, Potenza MN (2012). Diminished frontostriatal activity during processing of monetary rewards and losses in pathological gambling. Biol Psychiatry 71: 749-757.

Bermpohl F, Kahnt T, Dalanay U, Hägele C, Sajonz B, Wegner T et al (2010). Altered representation of expected value in the orbitofrontal cortex in mania. Hum Brain Mapp 31: 958-969.
Bourne C, Aydemir O, Balanza-Martinez V, Bora E, Brissos S, Cavanagh JT et al (2013). Neuropsychological testing of cognitive impairment in euthymic bipolar disorder: an individual patient data meta-analysis. Acta Psychiatr Scand 128: 149-162.

Caseras X, Lawrence NS, Murphy K, Wise RG, Phillips ML (2013). Ventral striatum activity in response to reward: differences between bipolar I and II disorders. Am J Psychiatry 170: 533-541.

Chandler RA, Wakeley J, Goodwin GM, Rogers RD (2009). Altered risk-aversion and risk-seeking behavior in bipolar disorder. Biol Psychiatry 66: 840-846.

Chen C-H, Suckling J, Lennox BR, Ooi C, Bullmore ET (2011). A quantitative meta-analysis of fMRI studies in bipolar disorder. Bipolar Disord 13: 1-15.

Dager AD, Anderson BM, Stevens MC, Pulido C, Rosen R, Jiantonio-Kelly RE et al (2013). Influence of alcohol use and family history of alcoholism on neural response to alcohol cues in college drinkers. Alcohol Clin Exp Res 37: E161-E171.

Di Nicola M, Tedeschi D, Mazza M, Martinotti G, Harnic D, Catalano V et al (2010). Behavioural addictions in bipolar disorder patients: role of impulsivity and personality dimensions. J Affect Disord 125: 82-88.

Everitt B, Robbins TW (2005). Neural systems of reinforcement for drug addiction: from actions to habits to compulsion. Nat Neurosci 8: 1481-1489.

Gabbay V, Ely BA, Li Q, Bangaru SD, Panzer AM, Alonso CM et al (2013). Striatum-based circuitry of adolescent depression and anhedonia. J Am Acad Child Adolesc Psychiatry 52: 628-641. e613.

Godlewska BR, Yip SW, Near J, Goodwin GM, Cowen PJ (2014). Cortical glutathione levels in young people with bipolar disorder: a pilot study using magnetic resonance spectroscopy. Psychopharmacol 231: 327-332.

Hafeman DM, Chang KD, Garrett AS, Sanders EM, Phillips ML (2012). Effects of medication on neuroimaging findings in bipolar disorder: an updated review. Bipolar Disord 14: 375-410.

Haldane M, Jogia J, Cobb A, Kozuch E, Kumari V, Frangou S (2008). Changes in brain activation during working memory and facial recognition tasks in patients with bipolar disorder with Lamotrigine monotherapy. Eur Neuropsychopharmacol 18: 48-54.

Hamilton MA (1960). A rating scale for depression. J Neurol Neurosurg Psychiatry 23: 56-61.

Hirschfeld RMA, Williams JBW, Spitzer RL, Calabrese JR, Flynn L, Keck PE Jr. et al (2000). Development and validation of a screening instrument for bipolar spectrum disorder: the mood disorder questionnaire. Am J Psychiatry 157: 1873-1875.

Hyatt CJ, Assaf M, Muska CE, Rosen RI, Thomas AD, Johnson MR et al (2012). Reward-related dorsal striatal activity differences between former and current cocaine dependent individuals during an interactive competitive game. PLoS One 7: e34917.

Insel T, Cuthbert B, Garvey M, Heinssen R, Pine D, Quinn $\mathrm{K}$ et al (2010). Research domain criteria (RDoC): toward a new classification framework for research on mental disorders. Am J Psychiatry 167: 748-751.

Jessup RK, O'Doherty JP (2011). Human dorsal striatal activity during choice discriminates reinforcement learning behavior from the gambler's fallacy. J Neurosci 31: 6296-6304.

Jia Z, Worhunsky PD, Carroll KM, Rounsaville BJ, Stevens MC, Pearlson GD et al (2011). An initial study of neural responses to monetary incentives as related to treatment outcome in cocaine dependence. Biol Psychiatry 70: 553-560.

Knutson B, Greer SM (2008). Anticipatory affect: neural correlates and consequences for choice. Phil Trans $R$ Soc B 363: 3771-3786.

Lawrence NS, Williams AM, Surguladze S, Giampietro V, Brammer MJ, Andrew C et al (2004). Subcortical and ventral prefrontal cortical neural responses to facial expressions distinguish patients with bipolar disorder and major depression. Biol Psychiatry 55: 578-587. 
Merikangas K, Akiskal H, Angst J, Greenberg P, Hirschfield R, Petukhova $\mathrm{M}$ et al (2007). Lifetime and 12-month prevalence of bipolar spectrum disorder in the national comorbidity survey replication. Arch Gen Psychiatry 64: 543-552.

Moses-Kolko EL, Price JC, Wisner KL, Hanusa BH, Meltzer CC, Berga SL et al (2012). Postpartum and depression status are associated with lower $\left[\left[{ }^{11} \mathrm{C}\right]\right.$ raclopride $\mathrm{BP}(\mathrm{ND})$ in reproductiveage women. Neuropsychopharmacol 37: 1422-1432.

Nusslock R, Almeida JRC, Forbes EE, Versace A, Frank E, LaBarbara EJ et al (2012). Waiting to win: elevated striatal and orbitofrontal cortical activity during reward anticipation in euthymic bipolar disorder adults. Bipolar Disord 14: 249-260.

Passarotti A, Sweeney J, Pavuluri M (2011). Fronto-limbic dysfunction in mania pre-treatment and persistent amygdala over-activity post-treatment in pediatric bipolar disorder. Psychopharmacol 216: 485-499.

Patel KT, Stevens MC, Meda SA, Muska C, Thomas AD, Potenza MN et al (2013). Robust changes in reward circuitry during reward loss in current and former cocaine users during performance of a monetary incentive delay task. Biol Psychiatry 74: 529-537.

Patton JH, Stanford MS, Barratt ES (1995). Factor structure of the barratt impulsiveness scale. J Clin Psychology 51: 768-774.

Peters J, Bromberg U, Schneider S, Brassen S, Menz M, Banaschewski T et al (2011). Lower ventral striatal activation during reward anticipation in adolescent smokers. Am J Psychiatry 168: 540-549.

Phillips MDM, Travis MDM, Fagiolini MDA, Kupfer MDD (2008). Medication effects in neuroimaging studies of bipolar disorder. Am J Psychiatry 165: 313-320.

Raven J, Court J, Raven J. Raven's Progressive Matrices. Oxford Psychologists Press: Oxford, UK, 1998.

Samanez-Larkin GR, Gibbs SE, Khanna K, Nielsen L, Carstensen LL, Knutson B (2007). Anticipation of monetary gain but not loss in healthy older adults. Nat Neurosci 10: 787-791.

Samanez-Larkin GR, Hollon NG, Carstensen LL, Knutson B (2008). Individual differences in insular sensitivity during loss anticipation predict avoidance learning. Psychol Sci 19: 320-323.

Schuckit MA (2012). Commentary on 'reduced subjective response to acute ethanol administration among young men with a broad bipolar phenotype'. Neuropsychopharmacol 37: 1797-1798.

Schuckit MA, Smith TL (1996). An 8-year follow-up of 450 sons of alcoholic and control subjects. Arch Gen Psychiatry 53: 202-210.

Sheehan D, Lecrubier Y, Sheehan K, Amorim P, Janavs J, Weiller E et al (1998). The Mini-International Neuropsychiatric Interview (M.I.N.I.INI): the development and validation of a structured diagnostic psychiatric interview for DSM-IV and ICD-10. J Clin Psychiatry 59(Suppl 20): 22-33.

Swann AC (2010). The strong relationship between bipolar and substance-use disorder. Ann NY Acad Sci 1187: 276-293.

Swann AC, Dougherty DM, Pazzaglia PJ, Pham M, Steinberg JL, Moeller FG (2005). Increased impulsivity associated with severity of suicide attempt history in patients with bipolar disorder. Am J Psychiatry 162: 1680-1687.

Tomasi D, Volkow ND (2013). Striatocortical pathway dysfunction in addiction and obesity: differences and similarities. Crit Rev Biochem Mol Biol 48: 1-19.

Valentin VV, O'Doherty JP (2009). Overlapping prediction errors in dorsal striatum during instrumental learning with juice and money reward in the human brain. J Neurophysiol 102: 3384-3391.

Volkow ND, Wang GJ, Telang F, Fowler JS, Logan J, Childress AR et al (2006). Cocaine craving cues and dopamine in dorsal striatum: Mechanism of craving in cocaine addiction. $J$ Neurosci 26: 6583-6588.

Vollstadt-Klein S, Wichert S, Rabinstein J, Buhler M, Klein O, Ende G et al (2010). Initial, habitual and compulsive alcohol use is characterized by a shift of cue processing from ventral to dorsal striatum. Addiction 105: 1741-1749.

Watson D, Clark LA, Tellegen A (1988). Development and validation of brief measures of positive and negative affect: the PANAS scales. J Pers Soc Psychol 54: 1063-1070.

Xu K, Liu H, Li H, Tang Y, Womer F, Jiang X et al (2014). Amplitude of low-frequency fluctuations in bipolar disorder: a resting state fMRI study. J Affect Disord 152-154: 237-242.

Yip SW, Chandler R, Rogers R, Mackay C, Goodwin G (2013). White matter alterations in antipsychotic- and mood stabilizernaive individuals with bipolar II/NOS disorder. Neuroimage Clin 3: $271-278$

Yip SW, Doherty J, Wakeley J, Saunders K, Tzagarakis C, de Wit H et al (2012). Reduced subjective response to acute ethanol administration among young men with a broad bipolar phenotype. Neuropsychopharmacol 37: 1808-1815.

Yip SW, Mackay CE, Goodwin GM (2014). Increased temporoinsular engagement in unmedicated bipolar II disorder: an exploratory resting state study using independent component analysis. Bipolar Disord (e-pub ahead of print; doi:10.1111/ bdi.12206).

Young RC, Biggs JT, Ziegler VE, Meyer DA (1978). A rating scale for mania: reliability, validity and sensitivity. Br J Psychiatry 133: 429-435.

Supplementary Information accompanies the paper on the Neuropsychopharmacology website (http://www.nature.com/npp) 